\title{
A Distributed Adaptive QoS MAC Protocol for Mobile Ad Hoc Networks
}

\author{
Mang Liao and Kai Liu \\ School of Electronics and Information Engineering, \\ Beihang University, Beijing 100191, China \\ Email: liaomang87@163.com, liuk@buaa.edu.cn
}

\begin{abstract}
This paper presents a distributed adaptive QoS medium access control (DAQ-MAC) protocol for mobile ad hoc networks. The protocol assigns nodes accessing channel in three phases: the prioritization phase, the contention phase and the transmission phase. The prioritizations of data packets are expressed by a few binary signed digits. In the prioritization phase, when binary signed digit is " 0 ", nodes sense the channel by a $T_{\text {slot, }}$ while binary signed digit is " 1 ", nodes send a forecast bursts (FB). Therefore, every node can differentiate the priorities by sending FB and sensing the channel. Thus only the nodes with higher priority packets can access in the contention phase. In the contention phase, only one node can access in the channel successfully without collisions by collision resolution algorithm. Simulation results show that with the number of the node increasing, the performance of the DAQ-MAC protocol is better than the HIPERLAN Type 1 (HIgh Performance Radio Local Area Networks) protocol in terms of throughput, average packets discard rate and average packet delay.
\end{abstract}

Keywords-medium access control (MAC); mobile ad hoc networks; quality of service (QOS); collision resolution

\section{INTRODUCTION}

A wireless ad hoc network does not require any infrastructure support in order to provide any type of communication among nodes [1]. MAC protocols are used for multiple nodes to share scarce wireless bandwidth in an orderly and efficient manner [2]. Recently, quality-of-service (QoS) is more and more important and necessary for real-time traffic such as voice and video [3].

Nowadays, many MAC protocols can guarantee QoS and most of them differentiate the packets priorities by adjusting the parameters about contention. And then they decrease collisions by collision avoidance algorithm. There are two ways of decreasing collisions assigned by network topology. One is randomize backoff strategy in distributed networks, such as IEEE 802.11e [4] protocol, DC-MAC [5] protocol and CMAC [6] protocol. While HiperLan/1 (HL/1) [7] protocol uses collision resolution to decrease collisions in distributed networks. Another one is that there is an access point (AP) in the networks and adopt polling way, such as PMAC [8]

This work was supported by the National Science Foundation for Innovative Research Groups of China under Grant 60921001, the National Outstanding Youth Science Foundation of China under Grant 60625102, the National 863 Program of China under Grant 2006AA12A103, and the "New Star in Blue Sky” Program of BUAA, China under Grant 221235. protocol.

IEEE 802.11e through enhanced distributed channel access (EDCA) [9] and DC-MAC protocol assign different priority by sensing the channel during different time. The nodes with higher priority packets can access in contention phase. In the contention phase, nodes access in the channel randomly. Furthermore when the nodes are happened collision, DC-MAC use cooperative nodes to retransmit. Because of collision avoidance of random access, when the nodes increase the probability that only one node is successful to access in the channel decrease.

CMAC protocol puts the contention phase into two periods. In the first period only nodes which transmit RT packets can send Black-burst (BB); the second period is for $\mathrm{BE}$ packets transmitted. Nodes which have transmitted $\mathrm{BB}$ first can access in the channel. The node that is successful into the channel uses cooperative nodes. The protocol does not consider transmission delay. A node may send BB before it senses $\mathrm{BB}$ which has been sent from other node. Therefore it will happen to collide.

$\mathrm{HL} / 1$ assigns the packets priorities into $\mathrm{H}$ classes (from 0 to H-1) by normalization MPDU residual lifetime (NMRC). Nodes which transmit $h_{i}\left(0 \leqslant h_{i} \leqslant H-1\right)$ priority packets sense the channel $h_{i} T_{\text {slot }}$ firstly. Only the nodes which sense the channel is idle can access to the contention phase. In the contention phase, the protocol decrease collisions by collision revolution. Therefore, when the nodes get more, performance of HL/1 will not get worse.

PMAC protocol assigns packets priorities into two kinds: RT packets and BE packets. The nodes which transmit RT packets are collected in the RT queue. The protocol assumes that there are two situations: the contention phase and the contention-free phase. In the contention phase it uses AP to poll the nodes in the RT queue; in the contention-free phase, poll the nodes in BE queue. Though the way of polling nodes by AP can solve the collision problem, the performance is relative to the number of nodes closely.

In order to achieve the standard of efficient MAC in multihop ad hoc networks, we provide a distributed adaptive QoS Multiple Access Control (DAQ-MAC) protocol. In the protocol, the packets priorities are expressed by a team of binary signed digits. When the binary digit is " 1 ", nodes send a FB signal. While binary digit is " 0 ", nodes sense the channel 
in one $\mathrm{T}_{\text {slot. }}$ Therefore we differentiate priorities by sending FB and sensing the channel. The nodes with the highest priority packets can access to the contention phase. Thus it can adapt QoS. In the contention phase, the protocol decreases collisions by collision revolution. When the nodes get more, the performance of the DAQ-MAC protocol keeps well.

The rest of this paper is organized as follows. In section II, we introduce the network model in DAQ-MAC protocol. In section III, the DAQ-MAC protocol will be presented in detail. The performance analysis of DAQ-MAC protocol will be shown in section IV. We discuss its performance evaluation in section $\mathrm{V}$ and conclude the paper in section VI.

\section{NETWORKS MODEL}

In the DAQ-MAC protocol, there are two zones: transmission zone and sensing zone in the networks. In transmission zone transmitting nodes and receiving nodes can receive others' packets exactly. In sensing zone nodes can sense packets from other nodes, but probably the packets could not be decoded exactly. Hence the range of sensing zone is more extensive than transmission zone. In the paper, we control the radius of sensing zone is twice than the radius of transmission zone by changing sensing threshold. Thereby it can avoid the problems of hidden terminal and exposed terminal.

\section{DAQ-MAC PROTOCOL}

In the DAQ-MAC protocol, channel access cycle consists of three phases: the prioritization phase, the contention phase and the transmission phase. If the channel is idle during a time interval $\mathrm{T}_{\mathrm{win}}$, nodes are allowed to start in prioritization phase. Otherwise, they have to wait for next access cycle.

The aim of the prioritization phase is to allow only nodes with the highest priority packets to participate in the contention phase. Firstly we assume that there are only two priority levels of the packets named real-time (RT) packets and best effort (BE) packets. The priority of RT packets is higher than the one of BE packets. In the prioritization phase only the nodes with RT packets send a FB while the nodes with BE packets sense the channel in one slot. We use this way to differentiate the prioritizations. If nodes with $\mathrm{BE}$ packets sense a FB signal, they will not access to the contention phase and have to wait for next access cycle. Therefore only the nodes with RT packets, if any, can access to the contention phase and thus the protocol can guarantee QoS.

The contention phase includes at most $n+m$ slots and a $\mathrm{T}_{\mathrm{obs}}$, and it consists of two phases: the sense period and the broadcast period. There are at most $\mathrm{n}$ slots in sense period and every node senses channel in J-1 slots. The nodes which sense the least slots idle can be admitted to the broadcast period, otherwise they have to wait for the next channel access cycle. $\mathrm{J}$ is a random variable with any kind of probability distribution such as truncated geometric distribution as follows

$$
p(J=j)= \begin{cases}(1-q) q^{j-1} \quad 1 \leq j<n \\ n-1\end{cases}
$$

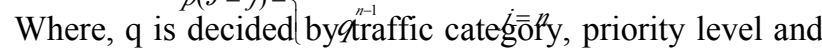
recent local average packet delay $\mathrm{D}$.

The broadcast period includes at most $\mathrm{m}$ slots and a $\mathrm{T}_{\mathrm{obs}}$ and each node broadcasts $\mathrm{k}$ FBs in that period, $0<\mathrm{k}<\mathrm{m}$. $\mathrm{m}$ is the maximum number of FBs for a node to send. The size of $\mathrm{k}$ follows some probability distribution related to packet delay. For instance, $\mathrm{k}$ can be selected between $\left[\mathrm{mD} / \mathrm{D}_{\max }, \mathrm{m}\right]$ in uniform distribution.

$$
f(k)=\left\{\begin{array}{cc}
\frac{1}{m-m D / D_{\max }} & m D / D_{\max }<k<m \\
0 & \text { others }
\end{array}\right.
$$

In the paper $D_{\max }$ stands for maximum packets delay and $\mathrm{D}$ stands for average packets delay. Therefore $\mathrm{k}$ can be selected appropriate value to improve packets of throughput and decrease average packet discard rate and average packet delay. After broadcasting k FBs, each node senses the channel for the duration of a $T_{\text {obs. }}$. If the channel is sensed busy, there is at least one node trying to access the channel and the node has to backoff. Otherwise, the node will win in the contention phase and start to transmit data packets in the transmission phase. After receiving the data packets correctly, the destination node will reply an acknowledgment (ACK) packet.

An example of the DAQ-MAC protocol is shown in Fig.1. In Fig.1, all the nodes must sense the channel during a time interval $\mathrm{T}_{\text {win. }}$. The channel is idle, so all the nodes can access to the prioritization phase. Node A, B and C have RT packets, so they send a FB in the prioritization phase, while node $\mathrm{D}$ and $\mathrm{E}$ have BE packets, so they sense the channel. Node D and E sense a FB from other nodes, so they cannot access to the contention phase and have to wait for the next cycle access. In the sense period of the contention phase node $C$ senses more $T_{\text {slot }}$ than node $A$ and $B\left(J_{C}>J_{A}=J_{B}\right)$ and it senses $F B$ from node $\mathrm{A}$ and node $\mathrm{B}$, so it can not be allowed to the broadcast period. Node $A$ sends more $F B s$ than node $B\left(K_{A}>K_{B}\right)$ in broadcast period and node $B$ senses the channel busy in $T_{o b s}$. Therefore node A wins the channel.

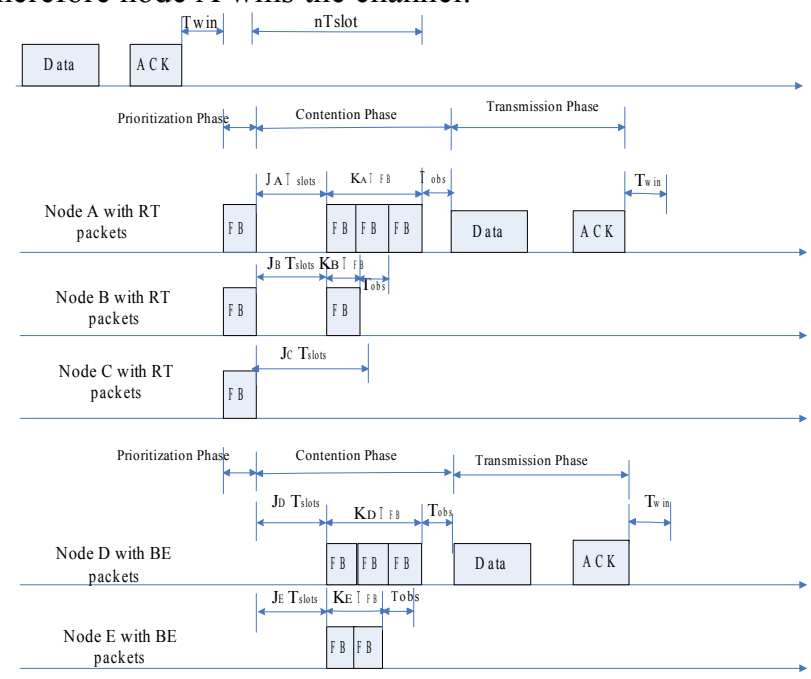

Figure 1.An example of DAQ-MACprotocol 


\section{PERFORMANCE ANALYSIS}

In the following analysis, we do not consider signal transmitting delay and send-received conversion time of transceiver and the delay of monitoring channel.

Because of higher demand of reliability of BE packets, if collisions are happened in the broadcast period of the contention phase, the nodes can return to this part seven times. However, when the nodes which have RT packets happen to collide in the broadcast period of the contention phase, the nodes have to be discarded. In the paper, we analyse three targets of the DAQ-MAC protocol: normalization throughput, average packets delay and average packets discard rate.

There are some parameters of the DAQ-MAC protocol following in the table I .

TABLE I Parameter Values of the DAQ-MAC Protocol

\begin{tabular}{ccc}
\hline maximum delay (/s) & $\mathrm{D}_{\max }$ & 0.1 \\
distributed parameter & $\mathrm{Q}$ & 0.8 \\
$\begin{array}{c}\text { arrive rate of every node } \\
\text { (packets/s) }\end{array}$ & $\lambda$ & 1 \\
Length & packets & 128 \\
(/bit) & Tslots & 9250 \\
Channel (Mb/s) & data transmission rate & 2 \\
& $\mathrm{~N}$ & 12 \\
& $\mathrm{M}$ & 12 \\
time (/us) & $\mathrm{T}_{\text {win }}$ & 268 \\
& $\mathrm{~T}_{\text {slot }}$ & 64 \\
& $\mathrm{~T}_{\mathrm{obs}}$ & 64 \\
& $\mathrm{~T}_{\mathrm{FB}}$ & 64 \\
& $\mathrm{~T}_{\mathrm{ack}}$ & 200 \\
& &
\end{tabular}

\section{1) Computation of Average Packet Discard Rate}

In the simulation, it is assumed that there are $\mathrm{N}$ nodes and message of every node generation process follows Poisson distribution. The Poisson probability distribution function of a random variable $\mathrm{X}$ is $p\{X=k\}=\frac{\lambda^{k} e^{-\lambda}}{k !}, k=0,1,2, \ldots, \lambda>0 \cdot \lambda_{0}$ stands for the arrival rate of every node message. Therefore the average arrival rate of all nodes can be computed as follows

$$
E(x)=N \lambda_{0}
$$

In the contention phase, we assume that $\mathrm{s}$ nodes which sense the channel during the least J-1 slots can access in the broadcast period of the contention phase. The probability distribution function of $\mathrm{S}$ is

$$
\operatorname{Pr}\{S=s\}=\left(\begin{array}{c}
N \\
s
\end{array}\right)\left(\sum_{j=0}^{n-1}\left((1-q) q^{j}\right)^{s}+\left(q^{n}\right)^{s}\left(1-q^{n}\right)^{N-s}\right)
$$

In the broadcast period, every node sends $\mathrm{K} \mathrm{FBs,} \mathrm{K}$ is selected between $\left[\mathrm{m}^{*} \mathrm{D} / \mathrm{D}_{\max }, \mathrm{m}\right]$ randomly, so the probability is $\frac{1}{m-m D / D_{\max }}$. When there are s nodes in the broadcast period, the probability that only one node can access in the transmission phase can be computed as follows

$$
P_{r}\{T=1 \mid S=s\}=s\left(\frac{1}{m-m D / D_{\max }}\right)\left(1-\frac{1}{m-m D / D_{\max }}\right)^{s-1}
$$

And then the probability that only one node is successful to use channel in the contention phase is

$$
\begin{aligned}
& P_{r}\{T=1\}=P_{r}\{S=s\}^{*} P_{r}\{T=1 \mid S=s\} \\
& =s\left(m-m D / D_{\max }\right)\left(\frac{1}{m-m D / D_{\max }}\right)\left(1-\frac{1}{m-m D / D_{\max }}\right)^{s-1} \\
& \left(\begin{array}{c}
N \\
s
\end{array}\right)\left(\sum_{j=0}^{n}\left((1-q) q^{j-1}\right)^{s}\left(q^{j}\right)^{N-s}\right)
\end{aligned}
$$

Let $\mathrm{P}_{\text {drop_RT }}$ and $\mathrm{P}_{\text {drop_BE }}$ be average discard rate of $\mathrm{RT}$ and BE packets, respectively. Therefore, $\mathrm{P}_{\text {drop_RT }}$ and $\mathrm{P}_{\text {drop_BE }}$ can be computed as

$$
\begin{gathered}
P_{\text {drop }{ }_{-} R T}=1-E(x) \operatorname{Pr}\{T=1\} \\
P_{d r o p_{-} B E}=1-\sum_{i=0}^{6} E(x) \operatorname{Pr}\{T=1\}(1-\operatorname{Pr}\{T=1\})^{i}
\end{gathered}
$$

\section{2) Computation of Average Packet Delay}

In the sense period of the contention phase, the average length that one node senses the least $\mathrm{J}-1$ slots is

$$
\sum_{j=0}^{n}\left[(1-q)+(1-q) q+\ldots+(1-q) q^{j-1}\right]=\sum_{j=0}^{n}\left(1-q^{j}\right)
$$

Therefore the average time lengths that s nodes sense the least J-1 slots can be expressed

$$
E\left[L_{s}\right]=\sum_{j=1}^{n}\left(1-q^{j}\right)^{s}-1
$$

And then the average data packets delay is

$$
D=T_{\text {slot }} * E\left[L_{s}\right]+T_{\text {win }}+T_{\text {slot }}
$$

Because $\mathrm{k}$ is selected between $\left[\mathrm{m} * \mathrm{D} / \mathrm{D}_{\max }, \mathrm{m}\right]$, the $\mathrm{k}$ slots lengths in the broadcast period can be computed as

$$
E\left[L_{B}\right]=\sum_{i=0}^{m-m D / D_{\max }}\left[m D / D_{\max }+i\right] * \frac{1}{m-m D / D_{\max }}
$$

So the total delay in the contention phase is

$$
E[L]=T_{\text {slot }} * E\left[L_{s}\right]+T_{F B} * E\left[L_{B}\right]+T_{o b s}
$$

Let $\mathrm{D}_{\mathrm{RT}}$ and $\mathrm{D}_{\mathrm{BE}}$ stand for average $\mathrm{RT}$ packets and $\mathrm{BE}$ packets delay, respectively. Therefore $\mathrm{D}_{\mathrm{RT}}$ and $\mathrm{D}_{\mathrm{BE}}$ can be computed as 


$$
\begin{gathered}
D_{R T}=T_{w i n}+T_{F B}+E[L] P_{r}\{T=1\}+L_{p k t} / R_{b}+T_{a c k} \\
D_{B E}=T_{w i n}+T_{F B}+\sum_{i=1}^{7} E[L] i \operatorname{Pr}\{T=1\}+L_{p k t} / R_{b}+T_{a c k}
\end{gathered}
$$

In the paper, $\mathrm{L}_{\mathrm{pkt}}$ stands for the every data packet lengths, and $\mathrm{R}_{\mathrm{b}}$ stands for the channel speed.

\section{3) Computation of Normalized Channel Throughput}

Let $\mathrm{S}$ express the normalized channel throughput. $\mathrm{S}$ is computed as

$$
S=\left(1-\mathrm{P}_{d r o p}\right) * E(x) * L_{p k t} / R_{b}
$$

\section{PERformance Evaluation}

\section{A. Simulation Environment}

The results of the DAQ-MAC protocol are simulated by $\mathrm{C}$ Language and compared with the results of HL/1 protocol. The simulation has modeled a network of $\mathrm{N}$ nodes randomly placed in an area $0.5^{*} 0.5 \mathrm{~km}^{2}$. The transmission radius $\mathrm{R}$ of a node is $0.1 \mathrm{~km}$. That is to say, the receivers can sense the situation of the channel in a 2-hop transmission range (2R). The data packets production of every node submit to Poisson distribution which is $p\{X=k\}=\frac{\lambda^{k} e^{-\lambda}}{k !}, k=0,1,2, \ldots, \lambda>0$. In the paper, we simulate and analyse the results from normalization throughput, average packet delay and average packet discard rate.

\section{B. Simulation Results}

In this subsection we assume that the channel is ideal and collision is the only reason that results in the failure of transmitting data packets. We do not care about the delay of transmission. By the parameters of Table I, we simulate the RT packets and BE packets results of DAQ-MAC protocol and compare them with the results from theoretical analysis as shown in Fig.2, 3 and 4.

Fig.2, 3 and 4 show that with the number of nodes increasing, the change of average packets delay, average packets discard rate and normalization throughput of theoretical analysis and simulation respectively. From Fig.2 we can see, with the number of nodes increasing, average RT packets delay will hold the line basically while average BE packets delay will increase. Furthermore average RT packets delay is less than average BE packets delay. The reason is that RT packets have higher priority and can be successful in the prioritization phase. So it can guarantee QoS. Fig.3 expresses that with the number of nodes increasing, average packets discard rate will increase and average RT packets discard rate is more than the one of average BE packets. The reason is that collision is happened easier when the nodes increasing, and that leads RT packets will be discarded more easily. In Fig.4, we can see with the number of nodes increasing, normalization throughput will increase. It illuminates that when nodes increase, the performance of DAQ-MAC protocol can keep well. The results of simulation are the same with the results of theoretical analysis in principle. Because of net topology and simulation environment, compared with the results of theoretical analysis, the results of simulation are a little descendant.

Performance results of the DAQ-MAC protocol and the HL/1 protocol are compared in Fig.5, 6 and 7.

Fig.5, 6 and 7 show the results of average packet delay, average discard rate and normalized throughput of the HL/1 protocol and the DAQ-MAC protocol respectively. As seen from Fig.5, with arrival rate increasing, the average data packets delay of the DAQ-MAC protocol and HL/1 protocol will hold the line and the result of HL/1 protocol is much more than the one of the DAQ-MAC protocol clearly. The reason is that in HL/1 protocol they use increasing the time of sensing channel to decrease the chance of node collision. In Fig. 6 and 7 , we can see performances of MAC protocol and HL/1 are the same at beginning. When arrive rate is over 0.7 , the performance of HL/1 will decrease, because the collision revolution algorithm of $\mathrm{HL} / 1$ relates to the number of the nodes closely, while DAQ-MAC protocol avoids this problem. Therefore the general performance of DAQ-MAC protocol is better than the one of HL/1.

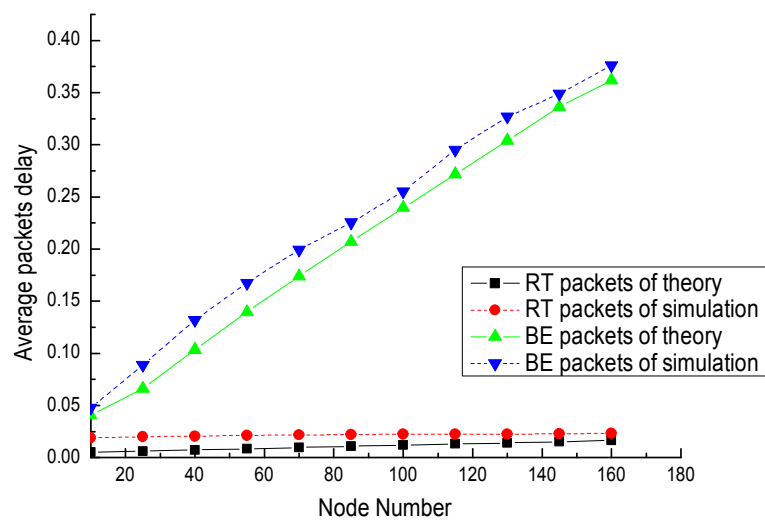

Figure 2. Average packet delay.

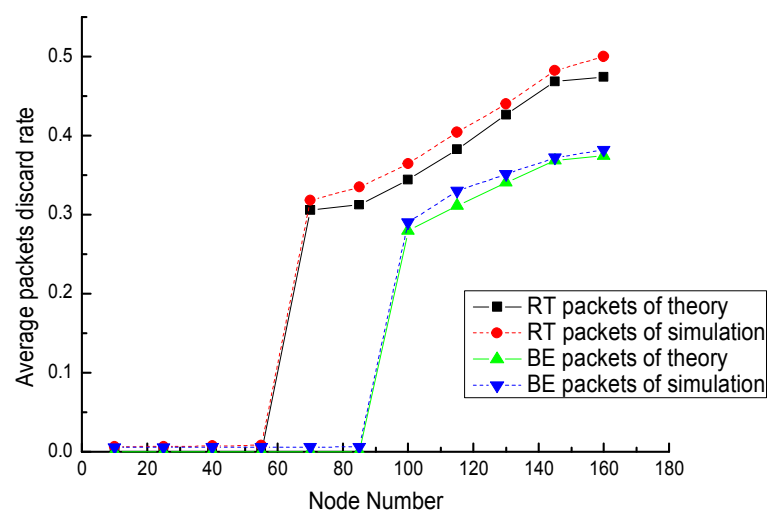

Figure 3. Average packet discard rate. 


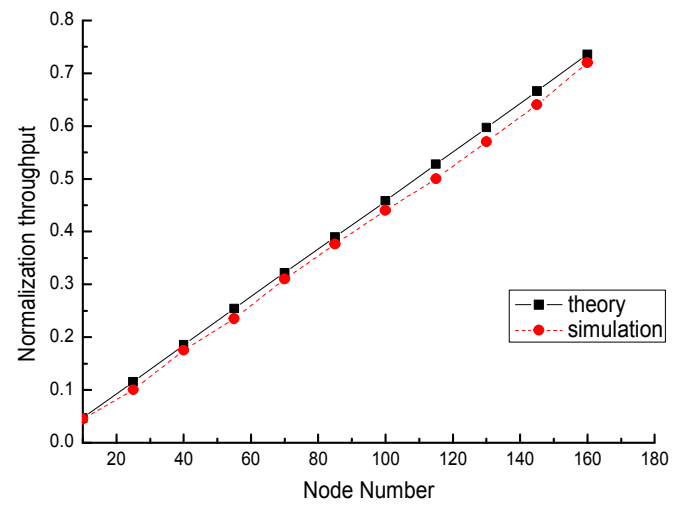

Figure 4. Normalized channel throughput.

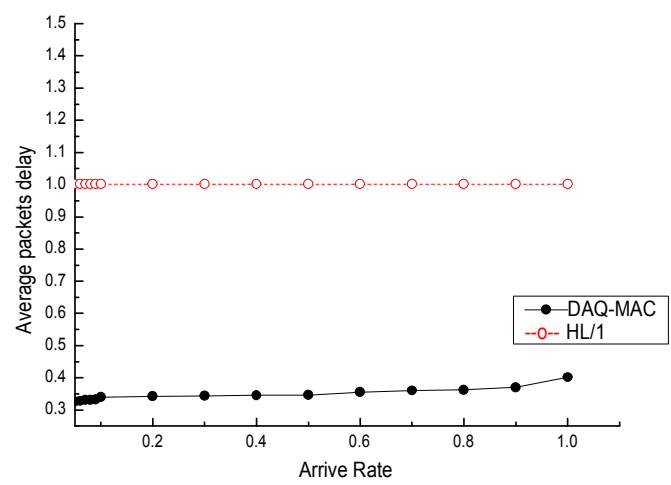

Figure 5. Average packet delay

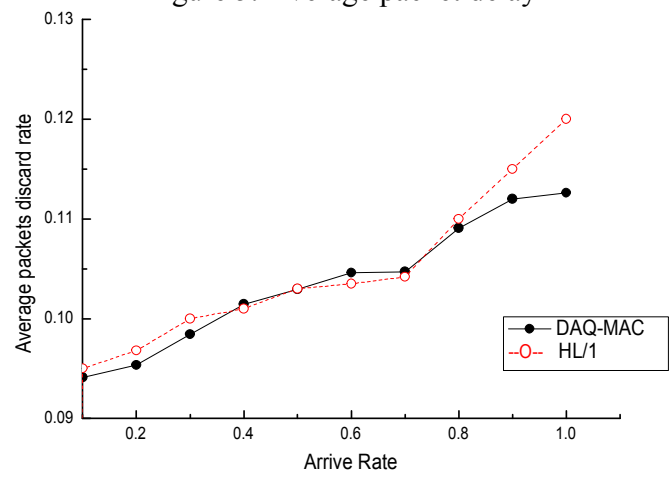

Figure 6. Average packet discard rate

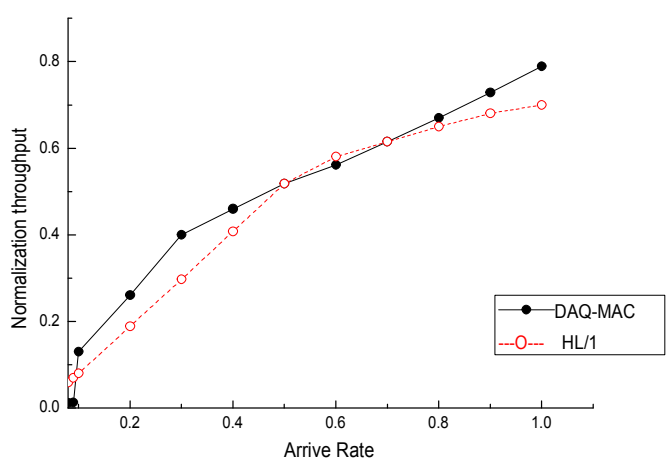

Figure 7. Normalization throughput

\section{CONCLUSION}

In this paper, we proposed a distributed adapted QoS MAC protocol for mobile ad hoc networks. In the protocol, the packet priority is expressed by a few binary signed digits and differentiated by sending FB and sensing the channel based on binary signed digits. Only the nodes with higher priority can access in the following phase. In the contention phase, nodes with the same priority packets use collision revolution algorithm to content the channel, and only one node which senses the least number of slots and broadcasts the most number of FBs can access in the channel, which leads to decrease of packets collisions and guarantees the QoS of data packets. Simulation results show that the performance of DAQ-MAC protocol is better than the one of $\mathrm{HL} / 1$ in terms of throughput, average packets discard rate and average packet delay.

\section{REFERENCES}

[1] Turki R and Mehmood R, “ Multimedia Ad Hoc Networks: Performance Analysis," Proc. IEEE. Computer Modeling and Simulation, pp. 561-566, Sep. 2008.

[2] Alizadeh-Shabdiz,f and Subramaniam, S,“ MAC layer performance analysis of multi-hop Ad Hoc networks," Proc. IEEE Conference, Global Telecommunications Conference, vol. 5, pp. 2781-2785, Jan 2005 .

[3] Yi Sheng Liu, Takawira, F and Hong Jun Xu, "Performance Analysis for a QoS-Aware Hybrid Token-CDMA MAC Protocol," IEEE $18^{\text {th }}$ International Symposum, pp. 1-6, Dec. 2007

[4] Peng F, Peng B and Qian D, "Performance analysis of IEEE 802.11e enhanced distributed channel access," IET Communication, vol. 4, no. 6, pp. 728-738, Apr. 2010.

[5] Tao Guo, Rolando Carrasco and Wai Lok Woo, "A Differentiated Cooperative MAC for QoS Enhancement in Wireless LANs," Personal, Indoor and Mobile Radio Communications, PIMRC 2008. IEEE $19^{\text {th }}$ International Symposium, Dec. 2008.

[6] Ho Ting Cheng, and Weihua Zhuang," QoS-Driven MAC-Layer Resource Allocation for Wireless Mesh Networks with Non-Altruistic Node Cooperation and Service Differentiation," IEEE Trans. Wireless Communications, vol. 8, no. 12, pp. 6089-6103, Dec. 2009.

[7] Giuseppe Anastasi, Luciano Lenzini, and Enzo Mingozzi," HIPER LAN/1 MAC Protocol: Stability and Performance Analysis," IEEE Journal on Selected Areas in Communications, vol. 18, no. 9, Sep. 2000.

[8] Jennifer Price, Pavan Nuggehalli and Tara Javidi," Incentive Compatible MAC-Layer QoS Design," Proc.IEEE Consumer Communications and Networking Conference (CCNC 08), pp. 586-590, Jan. 2008.

[9] Ilenia Tinnirello and Giuseppe Bianchi," Rethinking the IEEE 802.11e EDCA Performance Modeling Methodology," IEEE/ACM Trans. Networking, vol. 18 , no. 2, pp. 540-553, Oct. 2009 